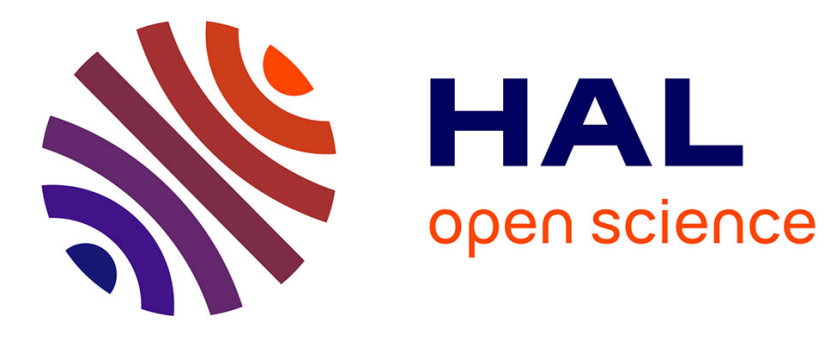

\title{
Wetting-Layer-Free AlGaN Quantum Dots for Ultraviolet Emitters
}

Guido Schifani, Thomas Frisch, Julien Brault, Philippe Vennegues, Samuel Matta, Maxim Korytov, Benjamin Damilano, Jean Massies, Jean-Noël Aqua

\section{- To cite this version:}

Guido Schifani, Thomas Frisch, Julien Brault, Philippe Vennegues, Samuel Matta, et al.. WettingLayer-Free AlGaN Quantum Dots for Ultraviolet Emitters. ACS Applied Nano Materials, 2020, 3 (5), pp.4054-4060. 10.1021/acsanm.9b02546 . hal-02883981

\section{HAL Id: hal-02883981 \\ https://hal.sorbonne-universite.fr/hal-02883981}

Submitted on 29 Jun 2020

HAL is a multi-disciplinary open access archive for the deposit and dissemination of scientific research documents, whether they are published or not. The documents may come from teaching and research institutions in France or abroad, or from public or private research centers.
L'archive ouverte pluridisciplinaire HAL, est destinée au dépôt et à la diffusion de documents scientifiques de niveau recherche, publiés ou non, émanant des établissements d'enseignement et de recherche français ou étrangers, des laboratoires publics ou privés. 


\title{
Wetting-Layer-Free AIGaN Quantum Dots for Ultraviolet Emitters
}

\author{
Guido Schifani, ${ }^{\dagger}$ Thomas Frisch, ${ }^{\dagger}$ Julien Brault,,,$\ddagger$ Philippe Vennéguès, ${ }^{\ddagger}$ Samuel \\ Matta, ${ }^{\ddagger}$ Maxim Korytov, ${ }^{\ddagger}$ Benjamin Damilano, ${ }^{\ddagger}$ Jean Massies, ${ }^{\ddagger}$ and Jean-Noël \\ Aqua*, \\ †Université Côte d'Azur, CNRS, Institut de Physique de Nice, Parc Valrose, 06108 Nice, \\ France \\ $\ddagger$ Université Côte d'Azur, CNRS, CRHEA, 06560 Valbonne, France \\ ФSorbonne Université, CNRS, Institut des Nanosciences de Paris, INSP, UMR 7588, 4 \\ place Jussieu, 75005 Paris, France \\ E-mail: Julien.Brault@crhea.cnrs.fr; aqua@insp.jussieu.fr
}

\begin{abstract}
We exhibit both experimentally and theoretically a novel growth mode for the epitaxy of AlGaN quantum dots (QD), where they are eventually produced without their usual surrounding wetting layer. If the generic evolution of $\mathrm{QD}$ is ruled by the elastic relaxation and capillary effects, evaporation occurs here on a time scale similar to that of growth. Using a dedicated surface diffusion model accounting for elasticity, wetting and anisotropy, we evidence numerically different kinetic regimes as a function of the evaporation flux, that rationalize the experimental outcome. The resulting QD are characterized by enhanced optical properties compared to the common QD with a wetting layer. These nanostructures are promising candidates for deep ultraviolet light emitting diodes.
\end{abstract}


keywords : ultra-violet emitters, epitaxy, quantum dots, wetting-layer, evaporation

Quantum dots are nowadays commonly and extensively used in different semi-conductor industrial and research devices, spanning e.g. from screen displays to UV emitters or indistinguishable photon sources. ${ }^{1-7}$ They may be produced by different technological routes but the technique best amenable to integration is hetero-epitaxy where they arise in the Stranski-Krastanov (SK) mode. ${ }^{8-10}$ In this case, the deposited film initially perfectly wets its substrate and grows layer-by-layer. Above some critical thickness $h_{c}$, it undergoes a morphological transition leading to QD that partially relax the elastic energy due to the misfit strain, and that remain separated by a thin wetting layer. This evolution is by its very nature out-of-equilibrium and requires the understanding of the crystal growth dynamics, a prerequisite to the control and application of these nanostructures in large-scale self-organized assemblies. ${ }^{11}$ In particular, QD fabricated in the SK mode have been recently used as the active region of UV light emitting diodes (LEDs) using Aluminium Gallium nitride (AlGaN) materials. ${ }^{5}$ These materials are seen as the next technology for UV sources in replacement of the mercury lamps, which bring issues of toxicity and recycling. In addition, LEDs present strong advantages such as low power consumption and long lifetimes. However, the external quantum efficiency of UV LEDs is still limited to typically of a few percent. ${ }^{12}$ This characteristic is, in part, due to the low internal quantum efficiency of the active region due to the high dislocation densities in AlGaN materials (usually above $10^{9} \mathrm{~cm}^{-2}$ ). One of the solutions proposed to circumvent this problem is to use QD instead of quantum wells as the active region: due to their dimensions at the nm scale, QD efficiently confine carriers and prevent them for recombining non radiatively on dislocations, leading to a weakly dependent photoluminescence intensity variation as a function of temperature compared to the QW case where carrier can diffuse towards dislocations as the temperature increases. ${ }^{13}$ However, due to the presence of a large internal electric field in GaN/AlGaN QD heterostructures (up to several $\mathrm{MV} / \mathrm{cm}$ ), the variation of the QD size (more precisely the QD height since the optical properties of GaN QDs are essentially governed by the confinement and the separation 
of electrons and holes, induced by the electric field, along the growth axis ${ }^{14}$ ), has a strong impact on their optical properties and in particular their wavelength emission due to the quantum confined Stark effect. ${ }^{15}$ Consequently, it is of importance to understand the main mechanisms leading to the QD formation in the SK mode.

We investigate here the effect of evaporation from the crystal to the high vacuum phase. It occurs significantly in $\mathrm{AlGaN}$ systems on accessible time scales and challenges the kinetic processes at work during crystal growth at the nanoscale. We find experimentally that a GaN film on an $\mathrm{Al}_{x} \mathrm{Ga}_{1-x} \mathrm{~N}$ substrate leads to quantum dots that coexist even though their wetting layer has disappeared. This finding is in contrast with the usual SK picture applicable to these systems, where QD coexist with a surrounding wetting layer. To rationalize this kinetic route, we use a dynamical model describing surface diffusion that accounts for evaporation, wetting and elasticity. Using parameters dedicated to AlGaN systems, it perfectly describes a dynamics leading to QD with a vanishing wetting layer. This preferential evaporation appears as a breakdown of the smooth evolution usually dictated by surface diffusion that kinetically competes here with evaporation. As QD offer an attractive way to localize charge carriers and hinder their diffusion to defects, we discuss the application of these nanostructures in deep ultraviolet devices.

\section{Experimental evidence}

The structures, see Fig. 1, were grown by molecular beam epitaxy using solid sources for Ga and $\mathrm{Al}$ elements and ammonia $\left(\mathrm{NH}_{3}\right)$ as the nitrogen source. Using sapphire as a substrate, an $\mathrm{Al}_{0.5} \mathrm{Ga}_{0.5} \mathrm{~N}$ (0001)-oriented layer (with a thickness of $800 \mathrm{~nm}$, ensuring the fabrication of an almost fully relaxed layer ${ }^{17,18}$ ) was first grown, followed by the fabrication of the GaN $\mathrm{QD}$ at a temperature of $800^{\circ} \mathrm{C}$. Initially, the growth mechanisms of $\mathrm{GaN}$ on $\mathrm{Al}_{0.5} \mathrm{Ga}_{0.5} \mathrm{~N}$ were investigated based on the evolution of the GaN layer morphology, which was monitored in situ by reflection high energy electron diffraction (RHEED): as presented in Fig. 2, the 

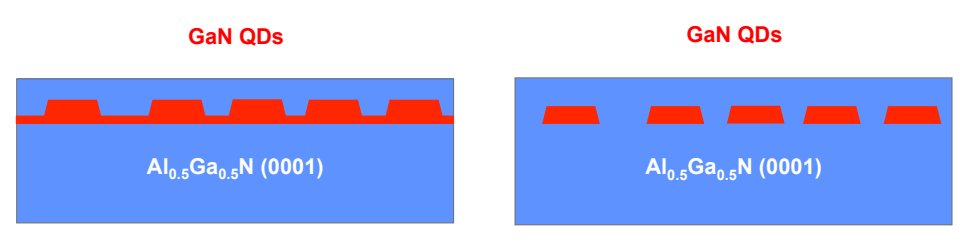

Figure 1: Structures of the samples investigated in this study: GaN QD, with (left) or without (right) a WL, were grown on $\mathrm{Al}_{0.5} \mathrm{Ga}_{0.5} \mathrm{~N}$. The samples were grown on (0001) sapphire substrates by molecular beam epitaxy (MBE) in a RIBER 32 reactor using solid sources for aluminium and gallium, and ammonia $\left(\mathrm{NH}_{3}\right)$ as nitrogen precursor. The $\mathrm{Al}$ content of the $\mathrm{Al}_{x} \mathrm{Ga}_{1-x} \mathrm{~N}$ layers was determined in-situ by reflection high-energy electron diffraction (RHEED) oscillations, ${ }^{16}$ and confirmed by energy dispersive X-ray spectroscopy in a scanning electron microscope (SEM).

intensity variation of the RHEED diagram as a function of time was recorded. As a general feature, the variation of the intensity, which corresponds here to the integrated intensity of two different regions of the RHEED diagram (Fig. 2(c)), can be directly correlated to the modification of the GaN layer going from a streaky pattern (Fig. 2(a)), produced by a two dimensional (2D) surface, to a spotty one (Fig. 2(b)), characteristic of a 3D morphology due to the formation of nanometer-sized islands (i.e. GaN QD). Actually, the formation of GaN QD using $\mathrm{NH}_{3}$ are observed after the deposition of GaN, providing that the growth interruption is performed under vacuum and that the deposited amount of GaN is superior to a critical thickness which depends on the lattice-mismatch value between the GaN and the $(\mathrm{Al}, \mathrm{Ga}) \mathrm{N}$ layers. ${ }^{19}$ The evolution of the RHEED intensity follows four stages which can be distinguished between different times (from $t_{0}$ to $t_{4}$ in Fig. 2(c)): i) in a first stage, i.e. between $t_{0}$ and $t_{1}$, a nominal 8 monolayers thickness of GaN is deposited on $\mathrm{Al}_{0.5} \mathrm{Ga}_{0.5} \mathrm{~N}$ and follows a 2D growth mode (Fig. 2(a)); ii) after the GaN growth, both Ga and $\mathrm{NH}_{3}$ fluxes are switched off (at $t_{1}$ ), and the 3D diffraction intensity increases gradually due to the formation of Bragg spots (while the specular spot intensity drops), reaching a maximum at $t_{2}$ and corresponding to the formation of QD; (iii) then, the RHEED intensity reaches a plateau and remains almost constant, between $t_{2}$ and $t_{3}$, indicating an invariance of the overall diffracted intensity, i.e. average diffracted QD volume; (iv) for times longer than 
$t_{3}$, a progressive decrease (increase) of the 3D surface Bragg spot intensity (2D surface specular spot) is observed, corresponding to a progressive transition from a $3 \mathrm{D}$ to a $2 \mathrm{D}$ surface morphology due to the evaporation of the GaN layer ${ }^{20}$ until the $\mathrm{Al}_{0.5} \mathrm{Ga}_{0.5} \mathrm{~N}$ surface is reached (at $t_{4}$ ). As a consequence, the QD morphology could be modified using different interruption times after the deposition of the GaN layer. ${ }^{21}$

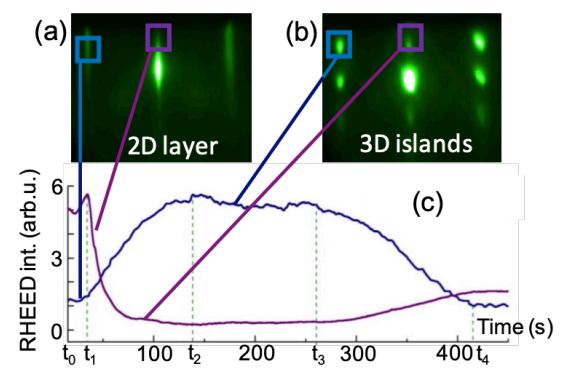

Figure 2: Reflection high energy electron diffraction (RHEED) diagrams of a GaN layer grown on $\mathrm{Al}_{0.5} \mathrm{Ga}_{0.5} \mathrm{~N}$ : (a) during the deposition of the $2 \mathrm{D}$ layer and (b) during the growth interruption under vacuum. (c) Integrated intensity variation as a function of time of two different regions of the RHEED diagram corresponding to the specular spot (purple) and the Bragg spot (dark blue) evolution.

Next, in order to further investigate the evolution of the GaN layer and QD morphology, two types of QD samples were fabricated using different interruption times (t) : 1) for $t$ in the vicinity of $t_{2}$ (i.e. $t \approx t_{2}$, sample A), 2) for $t$ longer than $t_{3}$ (i.e. for $t_{3}<t<t_{4}$, sample B). After the fabrication of the QD, a $30 \mathrm{~nm}$-thick capping layer of $\mathrm{Al}_{0.5} \mathrm{Ga}_{0.5} \mathrm{~N}$ was grown for the purpose of studying the QD morphology and optical properties. The QD morphologies of samples A and B were investigated by cross-sectional scanning transmission electron microscopy in high angle annular dark field mode (STEM-HAADF) as described in. ${ }^{21,22}$ Sample A consists of GaN QD formed after an annealing duration between $t_{2}$ and $t_{3}$ (see Fig. 2 and Fig. 3(a)) whereas sample B consists of GaN QD after an annealing duration between $t_{3}$ and $t_{4}$ (see Fig. 2 and Fig. 3(b)). In both cases QD have been capped with an $\mathrm{Al}_{0.5} \mathrm{Ga}_{0.5} \mathrm{~N}$ layer. HAADF images show $Z$-contrast with atomic columns intensities proportional to $Z^{\alpha}, Z$ being the average atomic number along the column and with $\alpha$ being between 1.6 and 2, GaN therefore appearing brighter than $\mathrm{Al}_{0.5} \mathrm{Ga}_{0.5} \mathrm{~N}$. GaN QD in sample 
A appears as sitting on a 2D GaN wetting layer (WL) with a thickness between 0.8 and $1 \mathrm{~nm}^{21}$ whereas sample B is made of a QD plane without a WL. ${ }^{22}$ The contrast fluctuations in the $\mathrm{Al}_{0.5} \mathrm{Ga}_{0.5} \mathrm{~N}$ layers are due to a phase separation mechanism described in detail in Ref. ${ }^{23}$ Consequently, it is found that the presence of an evaporation process of the GaN
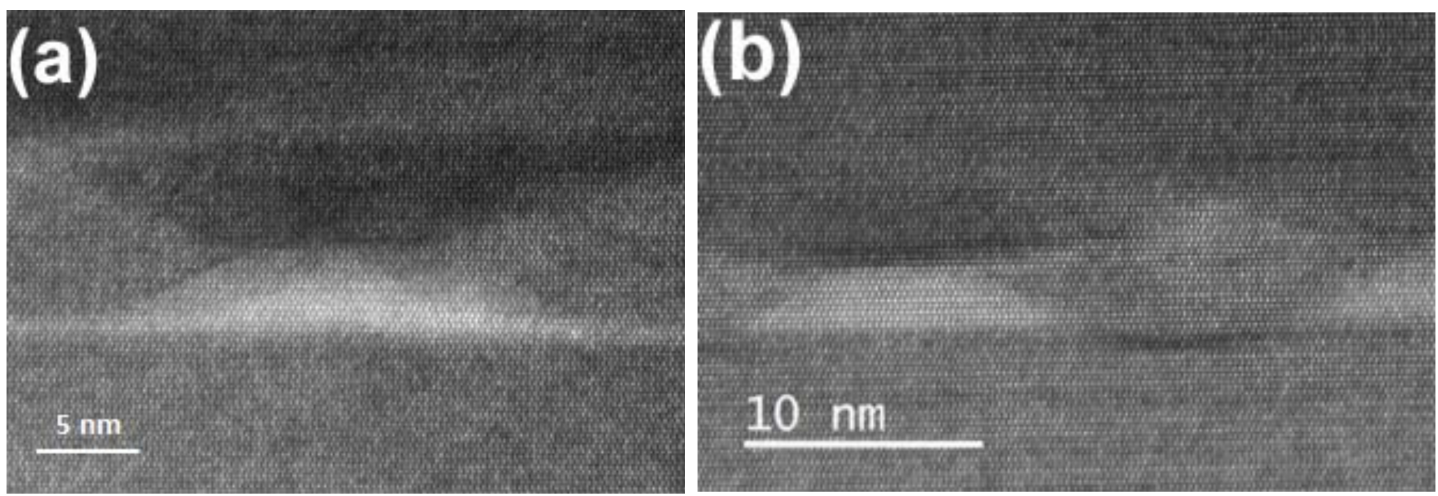

Figure 3: Cross section STEM-HAADF images of QDs along the [1-100] zone axis in sample $\mathrm{A}$ (a) and sample B (b). GaN appears brighter than $\mathrm{Al}_{0.5} \mathrm{Ga}_{0.5} \mathrm{~N}$ in such images. The contrast fluctuations in the $\mathrm{Al}_{0.5} \mathrm{Ga}_{0.5} \mathrm{~N}$ layers are due to a phase separation mechanism described in detail in. ${ }^{23}$

layer involving the formation of $\mathrm{QD}$ can lead to a specific regime where the initial presence of a WL can be suppressed.

It is noteworthy that the (1-103) facets forming the QDs are also observed when annealing under vacuum a 2D unstrained GaN layer in the same temperature range, i.e. when significant evaporation occurs. This clearly means that under vacuum the (0001) surface becomes less stable than the (1-103). As a consequence, the (0001) surface is rapidly replaced by a (1-103) facetted surface. Also, the evaporation rate of (1-103) facets is about a factor 10 lower than the one of (0001) surface (note that a still lower evaporation rate, i.e. a factor 100 , is found for (1-100) plane, the most stable for wurtzite GaN phase $\left.{ }^{24,25}\right)$. Note also that it was shown in previous studies that the QD shape and size could be modified with the growth of an AlGaN capping layer. ${ }^{22}$ However, for application purposes, it is necessary to use a capping layer in order to obtain a high radiative efficiency of the QD that is strongly reduced in the case of uncapped QD. ${ }^{26}$ This explains the use of a capping layer here on top 
of the QD to determine and compare their optical (photoluminescence) efficiency. Hence, an important experimental outcome of this study is that capped QD without a WL could be grown.
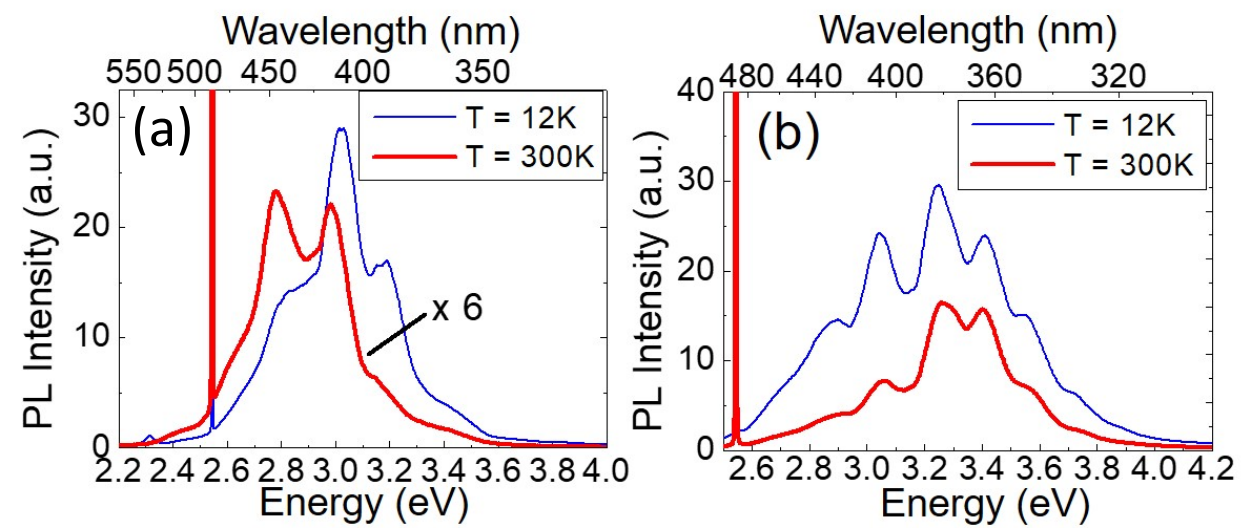

Figure 4: Comparison of the photoluminescence emission at $12 \mathrm{~K}$ and $300 \mathrm{~K}$ of GaN QD embedded in an (0001) matrix in the case of QD with (a) and without (b) a wetting layer. Note that the PL intensity of sample A at $300 \mathrm{~K}$ has been multiplied by a factor 6 .

We now turn to the consequence of this wetting-layer-free growth mode. A major result is found by studying and comparing the photoluminescence (PL) properties of samples A and $\mathrm{B}$ described above between $12 \mathrm{~K}$ and $300 \mathrm{~K}$. As shown in Fig. 4, the measurements are characterized by a broad modulated peak which originates from the QD emission. The modulation of the peak is due to interferences coming from the multi-layered structure of the samples, which are made of $\mathrm{AlGaN} / \mathrm{GaN} / \mathrm{AlN}$ layers grown on a sapphire substrate (similar to the growth process and conditions described in ${ }^{21}$ ). By comparing the PL integrated intensity of both samples, only a weak decrease is observed in the case of sample B, with a ratio of 1.6 between the measurement at $12 \mathrm{~K}$ and the one at $300 \mathrm{~K}$, whereas a stronger decrease is found for sample $\mathrm{A}$ with a ratio of 6 . These results are attributed to the role played by the WL in the QD properties: as the sample temperature is increased, thermally activated carriers initially injected into the QD are captured in the WL where they can then diffuse in the growth plane due to the reduction of confinement in the WL compared to the confinement in the QD. Consequently, the probability of carriers recombining non-radiatively 
with surrounding defects (i.e. mainly dislocations) increases, leading to a stronger reduction of the PL intensity compared to the case of QD without a WL. ${ }^{21}$ These results clearly show the important role of the WL in the non radiative processes and the interest in controlling the parameters at play to fabricate QD without a WL.

\section{Continuum evolution}

\section{Mass conservation}

We turn to the modelization of the dynamical evolution of hetero-epitaxial films. We use a continuum framework that describes the morphological evolution of a film deposited on a substrate. ${ }^{27}$ The film, lying in between $z=0$ and the free surface $z=h(x, y, t)$, evolves following the mass conservation ${ }^{28}$

$$
\frac{\partial h}{\partial t}=D \Delta \mu-F^{e v} \sqrt{1+\nabla h^{2}},
$$

where $F^{e v}$ corresponds to the evaporation flux, while $\mu=\partial\left(\mathcal{F}^{e l}+\mathcal{F}^{s}\right) / \partial h$ is the surface chemical potential given by the functional derivative of the elastic and capillary free energy $\mathcal{F}^{e l}$ and $\mathcal{F}^{s 29} 1$. Equation (1) includes both evaporation and surface currents due to surface chemical gradients. The crucial technical novelty here is the account of an explicit evaporation, that, generally speaking, may depend on the local orientation (different facets evaporate at different speeds). It introduces in the evolution equation (1) a source term in the right-hand side, that is function of the local slope. The influence of a similar yet different source term was analysed in ${ }^{27,28}$ with a deposition flux. In the seminal work of ${ }^{27}$ the deposition flux was shown to influence the critical film thickness for instability. It was found in ${ }^{28}$ that the resulting QD found in the latter stages of the instability are also dependent on the growth rate. Different regimes were found with a strong spatial correlation of QD at high

\footnotetext{
${ }^{1} \boldsymbol{\Delta}$ is the surface Laplacian equal to $\frac{\partial^{2}}{\partial x^{2}}+\frac{\partial^{2}}{\partial y^{2}}$ in the small slope approximation
} 
flux, that are always surrounded by a wetting layer. Yet, to the best of our knowledge, the influence of an evaporation flux has not been investigated. To describe AlGaN systems, we consider the substrate (0001) facet and the six (1-103) facets, with respectively the normal vectors $\mathbf{n}_{0}=\{0,0,1\}$ and $\mathbf{n}_{i}=\frac{1}{2}\left\{\cos \left(i \frac{\pi}{3}+\frac{\pi}{4}\right), \sin \left(i \frac{\pi}{3}+\frac{\pi}{4}\right), \sqrt{3}\right\}, i=1, \ldots 6$. In the following, we use an anisotropic evaporation flux

$$
\begin{gathered}
F^{e v}(\mathbf{m})=F_{0001}^{e v}\left\{1+\frac{1-p}{\pi} \sum_{j=1}^{6} \sum_{\sigma=0}^{1}(-1)^{\sigma} \ldots\right. \\
\left.\ldots \arctan \left[\eta\left(\left\|\mathbf{m}-\mathbf{m}_{j}\right\|-\frac{(-1)^{\sigma}}{\epsilon_{e}}\left\|\mathbf{m}_{j}\right\|\right)\right]\right\},
\end{gathered}
$$

with $\mathbf{m}=\left\{h_{x}, h_{y}\right\}, \mathbf{m}_{i}=2 / \sqrt{3}\left\{n_{i, x}, n_{i, y}\right\}, F_{0001}^{e v}$, the reference evaporation of the substrate, $p=F_{1-103}^{e v} / F_{0001}^{e v}$, and the parameters $\eta$ and $\epsilon_{e}$. We chose the generic parameters $A_{0}=0.2$, $\eta_{0}=10, \epsilon_{0}=0.1$, while $A_{i}=0.07, \eta_{i}=4, \epsilon_{i}=0.001$. These precise values are not important for the following results and chosen in order to reproduce the anisotropic properties of the AlGaN systems. We plot in Fig. 5 the evaporation flux $F^{e v}(\mathbf{m})$ used in the subsequent
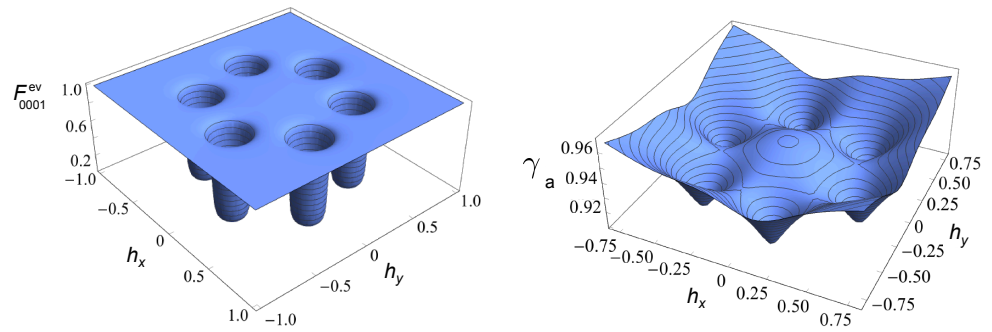

Figure 5: (left) Anisotropic evaporation flux used in the following simulations with a hexagonal anisotropy described in Eq. (2) with $p=\frac{1}{10}$; (right) Anisotropic surface energy described by Eq. (3).

analysis for a hexagonal anisotropy relevant for AlGaN systems. We use an anisotropy ratio $p=1 / 10$ that describes a tenfold decrease in evaporation between the substrate and the preferential (1-103) facets. This decrease corresponds to the experimental estimate that can be done from measurement on flat substrates. ${ }^{24}$ Though, as seen below, this precise value is not relevant for the following main results. In addition, the surface excess energy 
$\mathcal{F}^{s}=\int \gamma(h, \mathbf{n}) d^{2} S$ is decomposed as $\gamma=\gamma_{f}\left[1+\gamma_{a}(\mathbf{n})+\gamma_{w}(h)\right]$, with the thick-film (0001) reference $\gamma_{f}$, and the anisotropy and wetting contributions $\gamma_{a}$ and $\gamma_{w}$. ${ }^{30}$ We consider again a hexagonal symmetry for the surface energy anisotropy where

$$
\gamma_{a}=-\sum_{j=0}^{6} A_{j} \exp \left[-\eta_{j} \sqrt{1-\mathbf{n} . \mathbf{n}_{j}^{2}+\epsilon_{j}}\right]
$$

with the local normal $\mathbf{n}=\frac{1}{\sqrt{1+\nabla h^{2}}}\left\{-h_{x},-h_{y}, 1\right\}$. This functional leads to facet-like objets with a small regularization $\epsilon_{\alpha}$, even with a small amplitude $A_{\alpha}$, see Fig. 5 . In addition, we also consider wetting effects, that arise in nanometer-thick films; they originate from the excess contribution due to the presence of the film/substrate interface close to the film surface. In semi-conductors, we generically consider $\gamma_{w}(h)=c_{w} \exp \left(-h / \delta_{w}\right)$. Combined with elasticity and capillarity, wetting interactions enforce the existence of a critical thickness $h_{c}$ that is common of the Stranski-Krastanov (SK) mode : a flat film of thickness below $h_{c}$ does not evolve, while quantum dots separated by a wetting layer are found for thicknesses above $h_{c}$, see e.g. ${ }^{31,32}$. In the following, we used $c_{w}=0.01$ and $\delta_{w}=0.074$ that lead to $h_{c}=0.8 \mathrm{~nm}$ in the AlGaN system. Nevertheless, the following results do not depend on these precise values. Finally, the elastic energy in the system is due to the epitaxial misfit $m=\left(a^{f}-a^{s}\right) / a^{s}$ between the film $(f)$ and substrate $(s)$ lattice parameters $a^{f}\left(a^{s}\right)$. We use the elastic energy up to second order computed in the small slope approximation as given in. ${ }^{33}$ Its amplitude is related to the elastic energy density $\mathcal{E}_{0}=Y m^{2} /(1-\nu)$, where $Y$ and $\nu$ are the Young's modulus and Poisson's ratio. ${ }^{34}$

The natural length and time scales associated with the evolution equation (1) are $l_{0}=$ $\gamma_{f} / 2(1+\nu) \mathcal{E}_{0}$ and $t_{0}=l_{0}^{4} / D \gamma_{f}$ that can be derived by dimensional analysis. With the surface energy of GaN, $\gamma_{f}=1.89 \mathrm{~J} / \mathrm{m}^{2},{ }^{35,36}$ and with their typical elastic parameters ${ }^{2}$, one finds $l_{0}=5.4 \mathrm{~nm}$ for a GaN film on top of a $\mathrm{Al}_{0.5} \mathrm{Ga}_{0.5} \mathrm{~N}$ substrate. The setting of $t_{0}$ is more delicate as the effective diffusion coefficient $D$ is difficult to measure. To bypass this, we

\footnotetext{
${ }^{2}$ We used, $Y=300 \mathrm{GPa}, \nu=0.20, a_{G a N}=3.201 \AA, a_{A l N}=3.126 \AA$.
} 
compare the experimental and theoretical evolution of the roughness. The initial increase occurs experimentally on the time scale $\sim 100 \mathrm{~s}$, see Fig. 2 , and numerically on $\Delta t \sim 100 t_{0}$ so that we consider in the following $t_{0} \simeq 1 \mathrm{~s}$.

Note that in this modelization, we neglect effect of dislocations. Concerning the former, the main defects are threading dislocations which are propagating from the substrate/nitride layer interface up to the AlGaN surface where they create small surface depression. Regarding the influence of dislocations on the QD nucleation, it was shown in TEM studies that GaN QD can nucleate next to threading edge dislocations ${ }^{37}$ and we have also observed such features in low QD density samples. However, this feature has not been investigated here since the QD density is typically one order of magnitude larger than the threading dislocations density which means that at least around $90 \%$ of the QD formation is uncorrelated to the presence of threading dislocations. We also neglected the effects of interdiffusion at the substrate/film interface that could occur, but with an expected small influence on the surface diffusion on the pure GaN film.

\section{Kinetic regimes}

We investigate the dynamics dictated by Eq. (1) during evaporation of a film with an initial thickness $\bar{h}$. We typically find three different kinetic regimes, see Fig. 6 that are mainly function of the evaporation flux $F_{0001}^{e v}$ - that is proportional in real units to $F_{0001}^{e v} / D$. For a high evaporation flux (Fig. 6a), $F \gtrsim F_{c}^{\max }$, the instability (that develops on a time scale $t_{0}$ ) does not have time to occur significantly before the complete evaporation of the film (that occurs on a time scale $\bar{h} / F_{0001}^{e v}$. In this regime, the film mainly evaporates layer-by-layer. For intermediate flux (Fig. 6b), $F_{c}^{\min } \lesssim F \lesssim F_{c}^{\max }$, the morphological instability has time to develop during evaporation, QD arise, and may coarsen while matter evaporates. The main outcome is that in this regime, the wetting layer vanishes while QD are still present. In this case, evaporation is faster than the mass redistribution between islands, so that the thinner wetting layer disappears first at some locations where the bare AlGaN substrate is 
exposed, shown by gray areas in Fig. 6b. Finally, in the low evaporation flux regime (Fig. 6c), $F \lesssim F_{c}^{\text {min }}$, coarsening has ample time to occur before complete evaporation. Hence, islands form, coarsen and, as matter slowly evaporates, completely dissolve in the wetting layer that eventually evaporates layer-by-layer.

\section{Preferential evaporation of the WL}

We focus on the intermediate flux regime that is in strong correspondence with the experimental finding of QDs without a wetting layer. During the morphological evolution depicted in Fig. 6b, we find that the QD height first increases during the QD formation, then saturates while QDs slightly coarsen, and finally decreases as the system keeps on evaporating. The initial evolution is mostly dictated by surface diffusion and corresponds to the development of a morphological instability in its linear regime. It leads in the non-linear regime to facetted QDs surrounded by a WL, that slowly coarsen while their shape does not significantly evolve. As time goes by, evaporation starts to be visible and leads to the decrease of the QDs, and after some time, to the vanishing of the WL. During this evolution, once islands are formed, the wetting layer thickness is close but slightly higher than the SK critical thickness $h_{c}$, before it eventually falls below $h_{c}$ just before the first vanishing of the WL. Finally, it is worth noticing that the average film thickness decreases rather linearly during the entire process, even if the (0001) and (1-103) facets evaporate at different speeds.

To go further, we consider the time $\delta t^{e v}$ when the WL first vanishes, that is plotted in Fig. 7 as a function of $F_{0001}^{e v}$ for different evaporation anisotropy $p$. We find that (i) $\delta t^{e v}$ behaves typically as a power law $\delta t^{e v} \sim A / F^{\zeta}$, with $\zeta \simeq 1.1$; and (ii) it is bounded below by

$\delta t_{\min }^{e v}=\bar{h} / F_{0001}^{e v}$ corresponding to the evaporation of a flat film. An important conclusion of this Figure, is that the vanishing of the WL is not the result of the preferential evaporation of its orientation compared to the quantum dots facets (even though it does evaporate faster experimentally). Indeed, the same vanishing of the WL occurs when we consider an isotropic evaporation with $p=1$. In addition, there is very little quantitative difference in $\delta t^{e v}$ for 

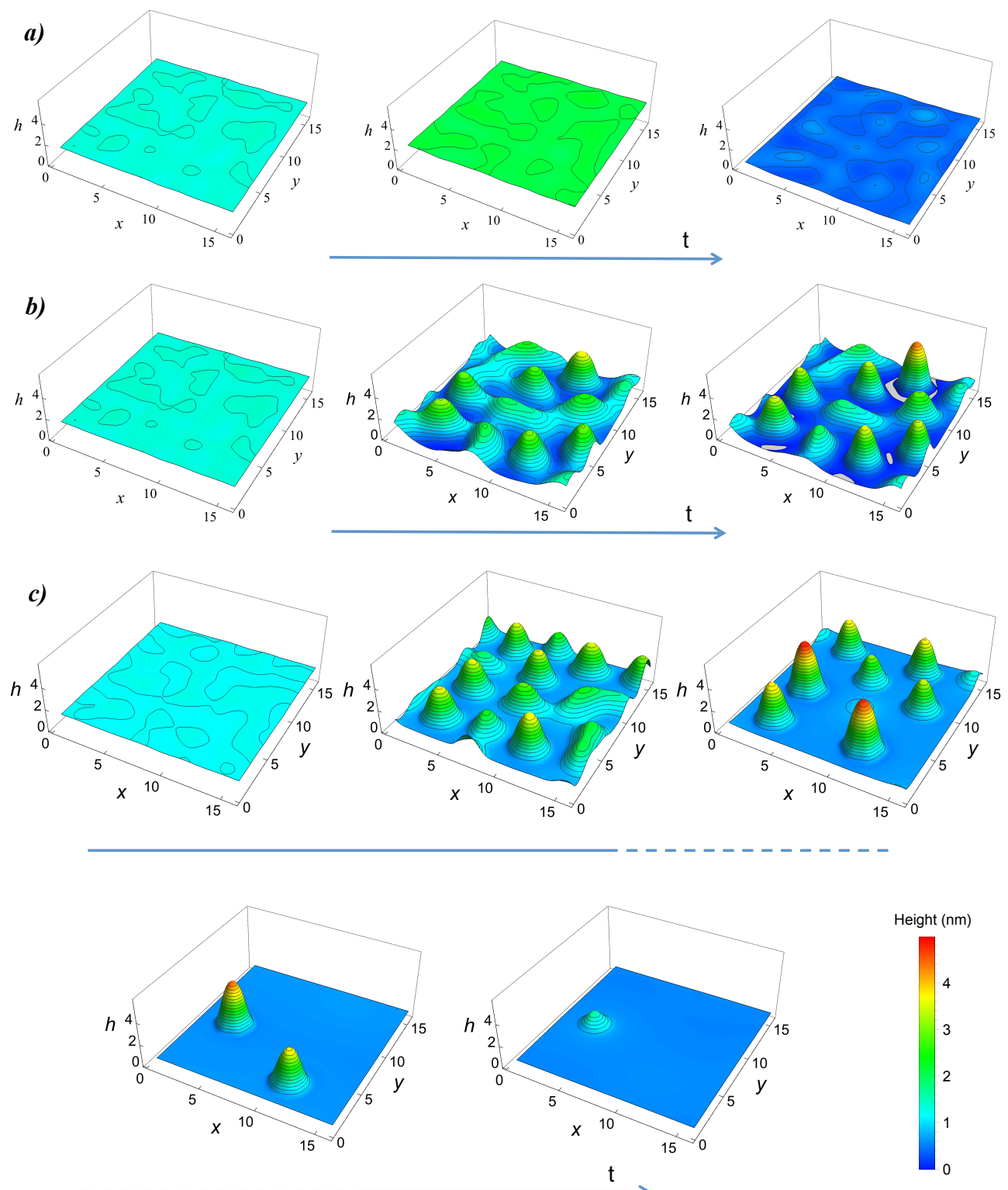

Figure 6: Evolution of a strained film following Eq. (1) for (a) a high evaporation flux, showing the initial morphological evolution followed by dissolution in the wetting layer while evaporation acts (the evaporation flux is $F_{0001}^{e v}=0.1$, while $\bar{h}=0.45(=2.4 \mathrm{~nm})$ and $\left.p=0.1\right)$; (b) an intermediate evaporation flux, with the growth of quantum dots, and their coarsening that leads to the preferential evaporation of the wetting layer : at some stage, evaporation leads to the exposition of the bare AlGaN substrate (gray areas) (the evaporation flux is $F_{0001}^{e v}=0.01$, while $\bar{h}=0.45(=2.4 \mathrm{~nm})$ and $\left.p=0.1\right) ;(\mathrm{c})$ a low evaporation flux, showing the initial growth of quantum dots followed by their coarsening and slow dissolution in the wetting layer while evaporation acts (the evaporation flux is $F_{0001}^{e v}=0.001$, while $\bar{h}=0.25$ $(=1.4 \mathrm{~nm})$ and $p=0.1$; in this case, we chose a slightly lower value of $\bar{h}$ in order to avoid a too big islands that undergo a shape transition beyond the scope of the article). In all the figures, the black level lines are separated by one monolayer. (lengths are given in nanometer) 


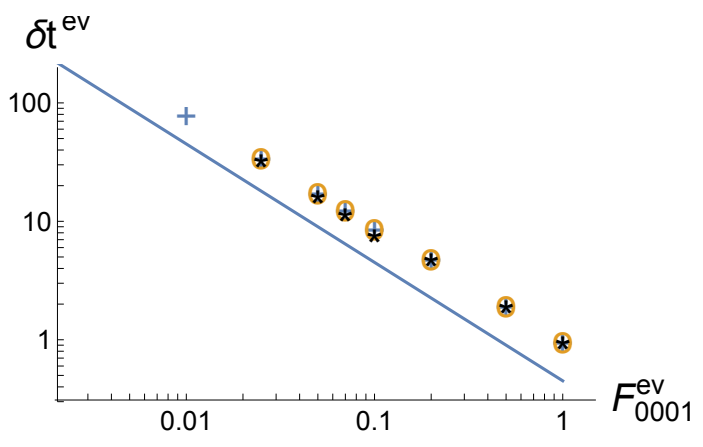

Figure 7: Time duration $\delta t^{e v}$ of the first vanishing of the WL as a function of the (0001) evaporation flux $F_{0001}^{e v}$ for a typical $\bar{h}=0.7$ (corresponding to $14 \mathrm{ML}$ ) and for (a) $p=0$ (blue + sign, no evaporation), (b) $p=0.1$ (orange circle) and (c) $p=1$ (black $*$ sign). The solid blue line is the lower bond $\delta t_{\min }^{e v}$ (see text).

the significantly different values of $p=0,0.1$ and even 1 , see Fig. 7. The vanishing of the WL results instead from the breakdown of the quasi-static evolution of the QD+WL system. All things considered, we can conclude that the preferential evaporation of the WL in the intermediate regime results from the fact that evaporation is too fast to allow a significant mass redistribution between the dots and the evaporating wetting layer, so that the system does not have time to heal the (thinner) evaporating wetting layer.

\section{Comparison with experiments}

The comparison between theory and experiments is delicate as some parameters are unknown precisely, e.g. concerning the diffusion coefficient. The experimental finding of QD without a WL is qualitatively in accordance with the simulations that lead to the vanishing of the $\mathrm{WL}^{3}$. We claim that this finding rationalizes the experimental initial vanishing of the WL. First, the experimental parameters are in the range of the intermediate regime. As explained above, if $D$ is delicate to measure, we can estimate $t_{0}$ by comparison of the initial increase of $w$, with the result $t_{0} \sim 1 \mathrm{~s}$ at $T=800^{\circ} \mathrm{C}$. In the experiments under study, the experimental evaporation of $2 \mathrm{D} \mathrm{GaN}$ is $0.17 \mathrm{ML} / \mathrm{s}$ while the evaporation of $\mathrm{GaN}(1-103) \mathrm{QDs}$ is $0.02 \mathrm{ML} / \mathrm{s}$,

\footnotetext{
${ }^{3}$ It should be noted that if we further pursue the simulation after the initial WL vanishing, the numerical code leads to a complete disappearance of the WL but, as the model is based on the assumption $h \geq 0$, this numerical finding is out of range of the model
} 


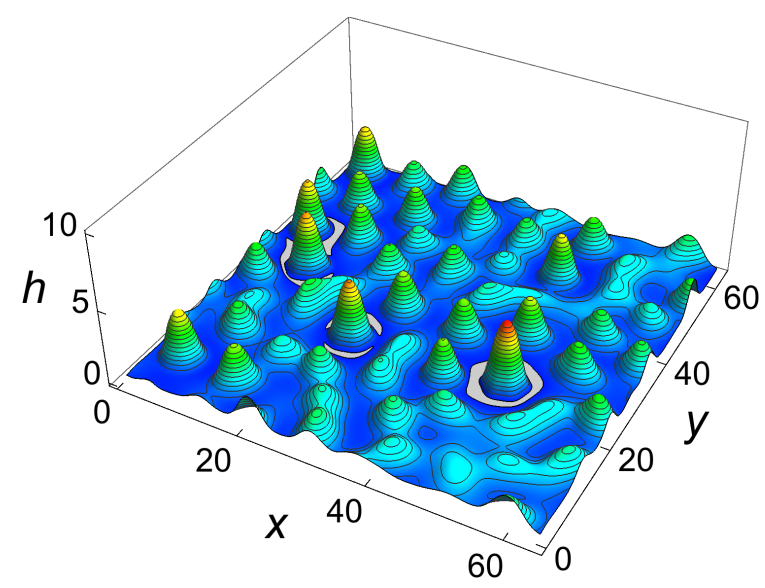

Figure 8: Simulation of the preferential evaporation of the wetting layer for parameters corresponding to experiments $F_{0001}^{e v}=0.010, \bar{h}=0.3$ and $p=0.12$, showing the vanishing of the wetting layer (gray areas) while quantum dots are already grown. (lengths are given in nanometer)

at this temperature, so that $F_{0001}^{e v}=0.010$ and $p=0.12$. With these experimental parameters, we performed the simulation of the film evolution under evaporation, see Fig. 8. We find in this case that the evolution does indeed lead to the evaporation of the WL while QDs are already formed, see Fig. 8. Thence, the experimental parameters are fully in the range where theory predicts a preferential evaporation of the WL. We consequently argue that the scenario of a competition between coarsening and evaporation rationalizes the experimental outcome of QD without their WL. We note that the process leading to the complete disappearance of the WL would require an appropriate model describing the triple line film/substrate/vacuum.

\section{Conclusion}

We have shown both experimentally and theoretically that a self-organized assembly of quantum dots separated by a wetting layer can lead during evaporation to dots without a wetting layer. We evidence this evolution in hetero-epitaxy of AlGaN systems and rationalize it thanks to a dedicated continuum dynamical model of surface diffusion. This model accounts for elasticity, surface energy anisotropy and wetting, and includes a facet-dependent evap- 
oration. For all the parameters investigated, it leads to the vanishing of the wetting layer on the long run, whether it has a preferential evaporation or not. But in some evaporation regime, it leads to quantum dots that are still present while the wetting layer has already evaporated. This study highlights the very kinetic nature of the usual geometries of the Stranski-Krastanov growth mode and the possibility to tune them. Furthermore, the resulting isolated quantum dots prove to have enhanced photoluminescence efficiencies compared to their usual counterparts with a wetting layer in the GaN/AlGaN system. These novel nanostructures open the path to new quantum-dot based devices that emit in the deep ultraviolet.

\section{Acknowledgement}

The authors thank D. Lefebvre, S. Vézian and B. Poulet for their invaluable technical assistance and scientific help. This work was supported by ANR Project (ANR-14-CE26-0025) "NANOGANUV".

\section{References}

(1) Lockwood, D. J. Towards Silicon-Based Photonic Integrated Circuits: The Quest for Compatible Light Sources. ECS Trans 2019, 89, 3 - 35.

(2) Zhang, X.; Li, H.-O.; Cao, G.; Xiao, M.; Guo, G.-C.; Guo, G.-P. Semiconductor quantum computation. Nat. Sci. Rev. 2018, 6, $32-54$.

(3) Choi, M. K.; Yang, J.; Hyeon, T.; Kim, D.-H. Flexible quantum dot light-emitting diodes for next-generation displays. NPJ Flexible Electronics 2018, 2, 10.

(4) Santana, T. S.; Ma, Y.; Malein, R. N. E.; Bastiman, F.; Clarke, E.; Gerardot, B. D. Generating indistinguishable photons from a quantum dot in a noisy environment. Phys. Rev. B 2017, 95, 201410(R). 
(5) Brault, J.; Matta, S.; Ngo, T.-H.; Rosales, D.; Leroux, M.; Damilano, B.; Al Khalfioui, M.; Tendille, F.; Chenot, S.; P., D.; Massies, J.; Gil, B. Ultraviolet light emitting diodes using III-N quantum dots. Mat. Sci. Semicond. Proces. 2016, 55, 95 - 101.

(6) Kneissl, M.; Kolbe, T.; Chua, C.; Kueller, V.; Lobo, N.; Stellmach, J.; Knauer, A.; Rodriguez, H.; Einfeldt, S.; Yang, Z.; Johnson, N. M.; Weyers, M. Advances in group IIInitride-based deep UV light-emitting diode technology. Semicond. Sci. Technol. 2010, 26, 014036 .

(7) Wang, L.; Wang, L.; Yu, J.; Hao, Z.; Luo, Y.; Sun, C.; Han, Y.; Xiong, B.; Wang, J.; Li, H. Abnormal Stranski-Krastanov Mode Growth of Green InGaN Quantum Dots: Morphology, Optical Properties, and Applications in Light-Emitting Devices. ACS Applied Materials \& Interfaces 2019, 11, 1228-1238.

(8) Stranski, I. N.; Krastanov, L. Zur Theorie der orientierten Ausscheidung von Ionenkristallen aufeinander. Ber. Akad. Wiss. Wien, Mater., Math.-Nat. Kl. IIb 1938, $146,797$.

(9) Markov, I. V. Crystal Growth for Beginners, 2nd ed.; WORLD SCIENTIFIC, 2003; p 564 .

(10) Aqua, J.-N.; Berbezier, I.; Favre, L.; Frisch, T.; Ronda, A. Growth and self-organization of SiGe nanostructures. Phys. Rep. 2013, 522, 59 - 189.

(11) Kiravittaya, S.; Rastelli, A.; Schmidt, O. G. Advanced quantum dot configurations. Rep. Prog. Phys. 2009, 72, 046502.

(12) Kneissl, M.; Seong, T. Y.; Han, J.; Amano, H. The emergence and prospects of deepultraviolet light-emitting diode technologies. Nat. Photon. 2019, 13, 233-244.

(13) Brault, J.; Rosales, D.; Damilano, B.; Leroux, M.; Courville, A.; Korytov, M.; Chenot, S.; Vennéguès, P.; Vinter, B.; DeMierry, P.; Kahouli, A.; Massies, J.; T., B.; 
Gil, B. Polar and semipolar GaN/Al0.5Ga0.5N nanostructures for UV light emitters. Semicond. Sci. Technol. 2014, 29, 084001.

(14) Bretagnon, T.; Lefebvre, P.; Valvin, P.; Bardoux, R.; Guillet, T.; Taliercio, T.; Gil, B.; Grandjean, N.; Semond, F.; Damilano, B.; Dussaigne, A.; Massies, J. Radiative lifetime of a single electron hole pair in GaN/AlN quantum dots. Phys. Rev. B 2006, 73, 113304.

(15) Brault, J.; Huault, T.; Natali, F.; Damilano, B.; Lefebvre, D.; Leroux, M.; Korytov, M.; Massies, J. Tailoring the shape of $\mathrm{GaN} / \mathrm{Al}_{x} \mathrm{Ga}_{1-x} \mathrm{~N}$ nanostructures to extend their luminescence in the visible range. J. Appl. Phys. 2009, 105, 033519.

(16) Grandjean, N.; Massies, J. GaN and $\mathrm{Al}_{x} \mathrm{Ga}_{1-x} \mathrm{~N}$ molecular beam epitaxy monitored by reflection high-energy electron diffraction. Appl. Phys. Lett. 1997, 71, 1816-1818.

(17) Huault, T.; Brault, J.; Natali, F.; Damilano, B.; Lefebvre, D.; Nguyen, L.; Leroux, M.; Massies, J. Blue-light emission from GaN $/ \mathrm{Al}_{0.5} \mathrm{Ga}_{0.5} \mathrm{~N}$ quantum dots. Appl. Phys. Lett. 2008, 92, 051911.

(18) Kahouli, A.; Kriouche, N.; Brault, J.; Damilano, B.; Vennéguès, P.; de Mierry, P.; Leroux, M.; Courville, A.; Tottereau, O.; Massies, J. GaN/ $\mathrm{Al}_{0.5} \mathrm{Ga}_{0.5} \mathrm{~N}$ (11-22) semipolar nanostructures: A way to get high luminescence efficiency in the near ultraviolet range. J. Appl. Phys. 2011, 110, 084318.

(19) Damilano, B.; Brault, J.; Massies, J. Formation of GaN quantum dots by molecular beam epitaxy using NH3 as nitrogen source. J. Appl. Phys. 2015, 118, 024304.

(20) Grandjean, N.; Massies, J.; Semond, F.; Karpov, S. Y.; Talalaev, R. A. GaN evaporation in molecular-beam epitaxy environment. Appl. Phys. Lett. 1999, 74, $1854-1856$.

(21) Brault, J.; Matta, S.; Ngo, T.-H.; Korytov, M.; Rosales, D.; Damilano, B.; Leroux, M.; Vennéguès, P.; Al Khalfioui, M.; Courville, A.; Tottereau, O.; Massies, J.; Gil, B. 
Investigation of $\mathrm{Al}_{y} \mathrm{Ga1}-y \mathrm{~N} / \mathrm{Al}_{0.5} \mathrm{Ga}_{0.5} \mathrm{~N}$ quantum dot properties for the design of ultraviolet emitters. Japan. J. Appl. Phys. 2016, 55, 05FG06.

(22) Korytov, M.; Huault, T.; Benaissa, M.; Neisius, T.; Brault, J.; Vennéguès, P. Effects of capping on GaN quantum dots deposited on $\mathrm{Al}_{0.5} \mathrm{Ga}_{0.5} \mathrm{~N}$ by molecular beam epitaxy. Appl. Phys. Lett. 2009, 94, 143105.

(23) Korytov, M.; Budagosky, J. A.; Brault, J.; Huault, T.; Benaissa, M.; Neisius, T.; Rouvière, J.-L.; Vennéguès, P. Mechanism of GaN quantum dot overgrowth by $\mathrm{Al}_{0.5} \mathrm{Ga}_{0.5} \mathrm{~N}$ : Strain evolution and phase separation. J. Appl. Phys. 2012, 111, 084309.

(24) Damilano, B.; Vézian, S.; Brault, J.; Alloing, B.; Massies, J. Selective Area Sublimation: A Simple Top-down Route for GaN-Based Nanowire Fabrication. Nano Lett. 2016, 16, $1863-1868$.

(25) Damilano, B.; Coulon, P.-M.; Vézian, S.; Brändli, V.; Duboz, J.-Y.; Massies, J.; Shields, P. A. Top-down fabrication of GaN nano-laser arrays by displacement Talbot lithography and selective area sublimation. Appl. Phys. Express 2019, 12, 045007.

(26) Kim, J.-H.; Elmaghraoui, D.; Leroux, M.; Korytov, M.; Vennéguès, P.; Jaziri, S.; Brault, J.; Cho, Y.-H. Strain- and surface-induced modification of photoluminescence from self-assembled $\mathrm{GaN} / \mathrm{Al} 0.5 \mathrm{Ga0} .5 \mathrm{~N}$ quantum dots: strong effect of capping layer and atmospheric condition. Nanotechnol. 2014, 25, 305703.

(27) Spencer, B. J.; Voorhees, P. W.; Davis, S. H. Morphological Instability in Epitaxially Strained Dislocation-Free Solid Films. Phys. Rev. Lett. 1991, 67, 3696-3699.

(28) Aqua, J.-N.; Frisch, T.; Verga, A. Ordering of strained islands during surface growth. Phys. Rev. E 2010, 81, 021605.

(29) Aqua, J.-N.; Frisch, T.; Verga, A. Nonlinear evolution of a morphological instability in a strained epitaxial film. Phys. Rev. B 2007, 76, 165319. 
(30) Aqua, J.-N.; Gouyé, A.; Auphan, T.; Frisch, T.; Ronda, A.; Berbezier, I. Orientation dependence of the elastic instability on strained SiGe films. Appl. Phys. Lett. 2011, 98, 161909.

(31) Leon, R.; Lobo, C.; Zou, J.; Romeo, T.; Cockayne, D. J. H. Stable and Metastable InGaAs/GaAs Island Shapes and Surfactantlike Suppression of the Wetting Transformation. Phys. Rev. Lett. 1998, 81, 2486-2489.

(32) Walther, T.; Cullis, A. G.; Norris, D. J.; Hopkinson, M. Nature of the StranskiKrastanow transition during epitaxy of InGaAs on GaAs. Phys. Rev. Lett. 2001, 86, 2381.

(33) Xu, X.; Aqua, J.-N.; Frisch, T. Growth kinetics in a strained crystal film on a wavy patterned substrate. J. Phys. : Condens. Matter 2012, 24, 045002, J.-N. Aqua and X. $\mathrm{Xu}$, Growth of quantum dots on pit-patterns, Surf. Sci. 2015, 20639.

(34) Aqua, J.-N.; Favre, L.; Ronda, A.; Benkouider, A.; Berbezier, I. Configurable Compliant Substrates for SiGe Nanomembrane Fabrication. Cryst. Growth Des. 2015, 15, 33993406.

(35) Einfeldt, S.; Kirchner, V.; Heinke, H.; Dießelberg, M.; Figge, S.; Vogeler, K.; Hommel, D. Strain relaxation in AlGaN under tensile plane stress. J. Appl. Phys. 2000, 88, 7029-7036.

(36) Ben Amar, A.; Faucher, M.; Brandli, V.; Cordier, Y.; Théron, D. Young's modulus extraction of epitaxial heterostructure AlGaN/GaN for MEMS application. Phys. Stat. Sol. (a) 2014, 211, 1655-1659.

(37) Rouvière, J. L.; Simon, J.; Pelekanos, N.; Daudin, B.; Feuillet, G. Preferential nucleation of GaN quantum dots at the edge of AlN threading dislocations. Appl. Phys. Lett. 1999, $75,2632-2634$. 


\section{Graphical TOC Entry}
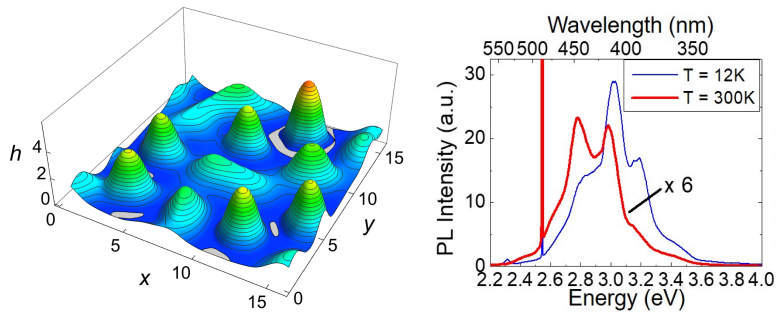